\title{
U-spin predictions of the transition magnetic moments of the electromagnetic decay of the $\Sigma^{*}(1385)$ baryons
}

\author{
D. Keller ${ }^{1, a}$ and K. Hicks ${ }^{2}$ \\ 1 University of Virginia, Charlottesville, Virginia 22901, USA \\ 2 Ohio University, Athens, Ohio 45701, USA
}

Received: 22 January 2013 / Revised: 25 March 2013

Published online: 3 May 2013

(C) The Author(s) 2013. This article is published with open access at Springerlink.com

Communicated by A. Ramos

\begin{abstract}
The transition magnetic moments for decuplet-to-octet baryon electromagnetic decays are calculated from the CLAS experimental results and are compared with calculations to first order in the $1 / N_{c}$ expansion of quantum chromodynamics (QCD) and new U-spin predictions. Using the U-spin predictions for the $\Sigma^{* 0} \rightarrow \Sigma^{0} \gamma$ and $\Sigma^{*+} \rightarrow \Sigma^{+} \gamma$ decays, the $S U(3)$-forbidden transition $\Sigma^{*-} \rightarrow \Sigma^{-} \gamma$ is obtained. In addition, the doubly strange baryon radiative decay $\Xi^{* 0} \rightarrow \Xi \gamma$ is predicted using U-spin.
\end{abstract}

\section{Introduction}

The measurement of baryon magnetic moments (and transition magnetic moments) provides a fundamental test of models based on QCD. Because the electromagnetic (EM) interaction is known, measurements of EM decay provide direct information on the wave function of the baryon [1]. For example, simple $S U(6)$ wave functions of baryons predict, in the context of a non-relativistic quark model, relations between baryon magnetic moments that are in remarkable agreement with experiment for ground-state baryons [2], yet are off by $30 \%$ or more when compared with the $\Delta \rightarrow N \gamma$ EM decay width [3]. The latter transition magnetic moment has been explained assuming that a pion "cloud" surrounds the nucleon [4]. This implies that the $S U(6)$ baryon wave functions are much too simplistic, at least for EM decays of baryons.

A better approach to interpreting the experimental measurements is to perfom a calculation based directly on quantum chromodynamics (QCD). One way to approach this problem is with lattice gauge theory. Calculations on the lattice for baryon transition magnetic moments were published nearly two decades ago [5], but these results do not agree with experiment and cannot properly account for the pion cloud because they used the quenched approximation [6]. Dynamical calculations on the lattice using full QCD are now routine, and transition magnetic moments for baryons have recently been determined for full QCD lattice calculations [7]. Unfortunately, such calcuations for strange baryons are not yet available.

\footnotetext{
a e-mail: dustin@jlab.org
}

An alternative to lattice QCD is to use the $1 / N_{c}$ expansion, where $N_{c}$ is the number of colors in QCD. In the real world, $N_{c}=3$, but theoretically one can explore the limit as $N_{c}$ becomes large, and develop a perturbative expansion in $1 / N_{c}$. Magnetic moments and transition magnetic moments have been calculated to first order in the $1 / N_{c}$ expansion by Jenkins and Manohar [8] and more recently beyond first order by Jenkins [9]. This more recent publication combines the $1 / N_{c}$ expansion with $S U(3)$ flavor symmetry breaking and isospin symmetry breaking. There are unknown constants in the expansions for the baryon magnetic moments, but the expansions can be combined such that, to first order, the unknown constants cancel in relations between baryons.

It is possible to group the quarks in terms of their charge, forming a different subgroup of $S U(3)$ in the exchange of $d$ and $s$ quarks, which is known as U-spin [10]. While U-spin is expected to be strongly broken by chiral symmetry, it respects charge symmetry.

The baryons are typically plotted as graphs of Ispin versus hypercharge. Plotted this way, particles of nearly equal mass come in horizontal rows. Alternatively, this graph can be rotated by $60^{\circ}$, giving U-spin versus charge, as shown in fig. 1. In this case, particles of equal charge form horizontal rows. This way of representing the low-mass baryons is useful when considering electromagnetic transitions, and in particular radiative decays of baryons. Put simply, $S U(3)_{f}$ can be written in terms of its subgroups as $S U(2)_{I} \times U(1)_{Y}$ or equivalently as $S U(2)_{U} \times U(1)_{Q}$.

The EM transition magnetic moments of the strange sector are of particular interest because they can be useful in determining the degree of $S U(3)$ symmetry breaking. 


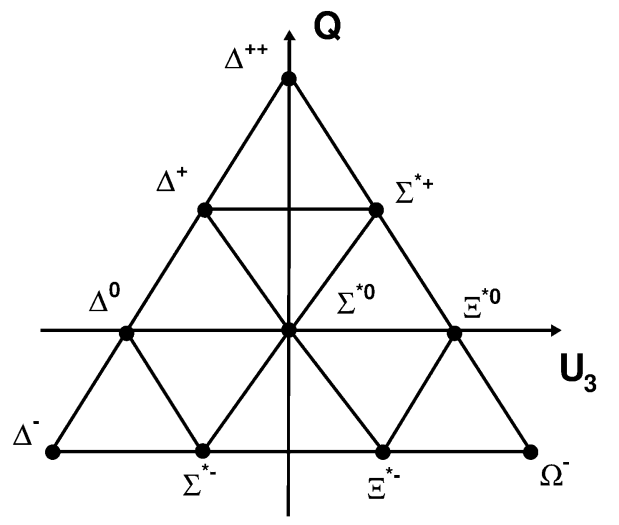

Fig. 1. Baryon decuplet, plotted for U-spin multiplets with charge $(Q)$ versus $\mathrm{U}$-spin $\left(U_{3}\right)$.

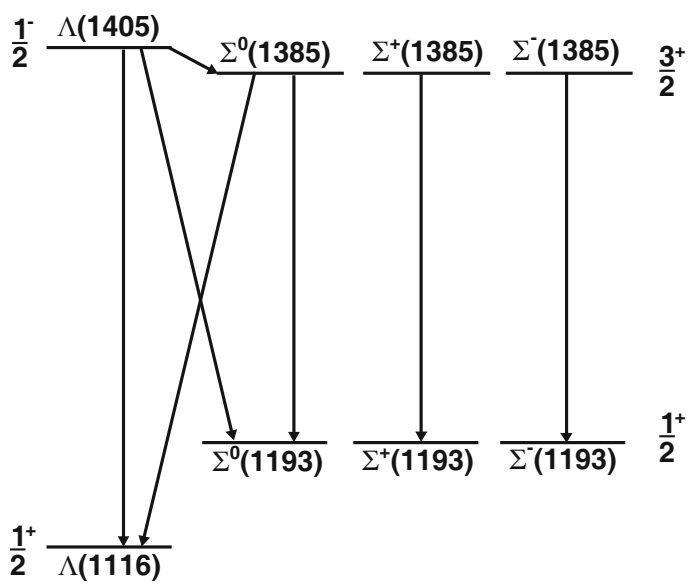

Fig. 2. Radiative-decay scheme for the $\Sigma(1385)$. Each charged decuplet-to-octet transition is shown side by side. The transition on the far right is U-spin forbidden.

U-spin symmetry forbids radiative decays of the $\Sigma^{*-}$ decuplet baryon. Since the photon is a charge singlet with $U=0$, a non-zero transition magnetic moment can reveal the degree to which U-spin symmetry is broken. This is easily understood by simply writing the $S U(6)$ wave functions for these baryons and inserting the $M 1$ operator between initial and final states,

$$
\left\langle\Sigma_{S U(6)}^{-}\left|\sum_{q} \frac{Q_{q}}{2 m_{q}} \sigma_{q} \cdot\left(\mathbf{k} \times \epsilon^{* \lambda}\right)\right| \Sigma_{S U(6)}^{*-}\right\rangle=0 .
$$

Here the sum is over all $q$ constituent quarks, $m_{q}, \sigma_{q}$ and $Q_{q}$ are the mass, spin vector and charge of the $q$ quark, $\mathbf{k}$ is the propagation direction, and $\epsilon^{* \lambda}$ is the polarization vector. In the same way the transition operator for the $\Sigma^{*+}$ gives a non-zero amplitude. U-spin invariance implies a large difference in the radiative decay widths of the $\Sigma^{*-}$ and $\Sigma^{*+}$. Figure 2 shows the EM decay scheme for each charged decuplet to octet transition for the $\Sigma$ baryon.

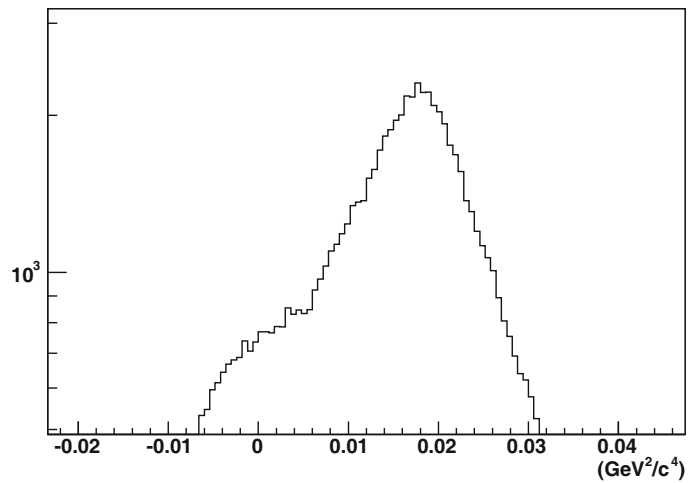

Fig. 3. The missing-mass squared spectrum from $\pi_{1}^{+} \pi^{-} \pi_{2}^{+} n$. Constraints have been applied to show the mass squared of the EM and $\pi^{0}$ decays from $\Sigma^{*+}$.

Using recent CLAS measurements of the branching ratio of $\Sigma^{*+} \rightarrow \Sigma^{+} \gamma$, we obtain its transition magnetic moment. This result can be compared with predictions from the $1 / N_{c}$ expansion or predictions from U-spin symmetry using a previous experimental [11] result for the decay width of $\Sigma^{* 0} \rightarrow \Lambda \gamma$. Then using the U-spin predictions for the $\Sigma^{* 0} \rightarrow \Sigma^{0} \gamma$ and $\Sigma^{*+} \rightarrow \Sigma^{+} \gamma$ decays, the $S U(3)$ forbidden [10] transition $\Sigma^{*-} \rightarrow \Sigma^{-} \gamma$ is obtained, providing an estimate of $S U(3)$ symmetry breaking in the baryon decuplet.

\section{Electromagnetic decay branching ratio}

The measurement was carried out with the CLAS detector [12] at the Thomas Jefferson National Accelerator Facility, using the g11a data set which used a real photon beam produced by a $4.019 \mathrm{GeV}$ electron beam. This resulted in a photon energy range of $1.6-3.8 \mathrm{GeV}$. The photon energy was obtained from a magnetic spectrometer, the tagger, leading to an energy resolution of the production photon of $\sim 0.1 \%$. The center of a $40 \mathrm{~cm}$ long liquidhydrogen target was placed $10 \mathrm{~cm}$ upstream from the center of CLAS. The trigger required two charged particles in coincidence with the tagged electron leading to 20 billion triggers in the CLAS run period. The approximate integrated luminosity for the CLAS g11a run period used analysis and quoted results was $70 \mathrm{pb}^{-1}$.

The reaction studied in CLAS was $\gamma p \rightarrow$ $K^{0} \Sigma^{*+}(1385)$, where the $K^{0}$ decays to $K_{s}^{0} \rightarrow \pi^{+} \pi^{-}$and the $\Sigma^{*+}$ decays to $\Sigma^{+} \pi^{0}$ or $\Sigma^{+} \gamma$. Finally, the $\Sigma^{+}$decays to $\Sigma^{+} \rightarrow n \pi^{+}$. All charged particles were detected via the drift chambers while the neutron was reconstructed using the CLAS electromagnetic calorimeter.

Various kinematic constraints were applied until the $\Sigma^{*+}$ EM decay signal became visable. The undetected final-state particle, either a $\pi^{0}$ from strong decay or a $\gamma$ from EM decay, was found from the conservation of momentum and energy. The missing-mass squared spectrum from $\pi_{1}^{+} \pi^{-} \pi_{2}^{+} n$ with constrains to show the EM peak is shown in fig. 3 . 
The strong and EM decays were resolved using kinematic fitting. The acceptance corrected counts of the EM and $\pi^{0}$ signals accounted for leakage of all possible background contributions. The final result is a measurement of the decay width ratio from [13],

$$
\left.R=\frac{\Gamma\left(\Sigma^{*+} \rightarrow \Sigma^{+} \gamma\right)}{\Gamma\left(\Sigma^{*+} \rightarrow \Sigma^{+} \pi^{0}\right)}=11.95 \pm 2.21 \text { (stat. }\right)_{-1.21}^{+0.53}(\text { sys. }) \%
$$

\section{Transition magnetic moments}

The transition magnetic moment of the EM decay of the $\Sigma^{*+}$ can be calculated using the experimental results from eq. (2), the full width of the $\Sigma^{*+}$ decay of $35.8 \pm 0.8 \mathrm{MeV}$ and the known branching ratio for $\Sigma^{*+} \rightarrow \Sigma^{+} \pi^{0} 5.85 \pm$ $0.75 \%$ from the Particle Data Group [14], along with $R$ above. The resulting EM decay width is

$$
\Gamma_{\Sigma^{*+} \rightarrow \Sigma^{+\gamma}}=250 \pm 57 \text { (stat.) }{ }_{-41}^{+34} \text { (sys.) } \mathrm{keV} .
$$

The baryon radiative decays $B\left(\frac{3}{2}^{+}\right) \rightarrow B^{\prime}\left(\frac{1}{2}^{+}\right) \gamma$ are $M 1$ and $E 2$ electromagnetic transitions with the spin flip of one of the quarks in baryon $B$. The $E 2$ amplitudes are very small and are assumed negligable. Using the dominance of the $M 1$ electromagnetic transition, the conversion of the experimental decay width to the magnetic moment becomes [5]

$$
\mu_{\Sigma \Sigma^{*+}}=\sqrt{\frac{2 M_{p}^{2} \Gamma_{\Sigma^{*+} \rightarrow \Sigma^{+} \gamma}}{\alpha p_{\gamma}^{3}}}=(3.22 \pm 0.45) \mu_{N},
$$

where $p_{\gamma}=0.180 \mathrm{GeV}$ is the momentum of the photon, $M_{p}$ is the proton mass, $\alpha$ is the fine structure constant, and $\mu_{N}$ is the nuclear magneton. Using eq. (10) adds $\sim$ $0.1 \%$ uncertainty to the value of the magnetic moment due to the exclusion of the $E 1$ contribution. This uncertainty is estimated from the work in the three flavor generalization of the Skyrme model [15]. A similar extraction for the transition magnetic moment is found for $\Sigma^{* 0} \rightarrow \Lambda \gamma$. A measurement of the EM decays widths for the other two channels, $\Sigma^{* 0} \rightarrow \Sigma^{0} \gamma$ and $\Sigma^{*-} \rightarrow \Sigma^{-} \gamma$, has not yet been established.

\section{The U-spin predictions}

It is possible, using the U-spin $S U(3)$ multiplet representation, to obtain a prediction for the ratio of the $\Delta^{0} \rightarrow n \gamma$ partial width to the $\Sigma^{* 0} \rightarrow \Lambda \gamma$ partial width. This implies that the experimental partial width of the $\Delta^{0} \rightarrow n \gamma$ reaction can be used with the U-spin Clebsh-Gordon coefficients along with the corresponding phase-space factors to obtain the expected partial width of the $\Sigma^{* 0} \rightarrow \Lambda \gamma$. In the strict limit of $S U(3)$ symmetry, U-spin is conserved for all processes. Only radiative transition between states with the same value of U-spin can occur within this limit.
In U-spin space, the unitary rotation in the $S U(2)$ symmetry space can be used to discribe the mixing between the neutral ground-state $S U(3)$ multiplet members $\Lambda$ and $\Sigma^{0}$ such that,

$$
\begin{aligned}
\left|\Sigma_{U}^{0}\right\rangle & =\frac{1}{2}|U=1\rangle+\frac{\sqrt{3}}{2}|U=0\rangle \\
\left|\Lambda_{U}\right\rangle & =\frac{1}{2}|U=0\rangle-\frac{\sqrt{3}}{2}|U=1\rangle .
\end{aligned}
$$

The amplitude requires the Clebsch-Gordon coefficients, which can be found by contraction of the initial-excitedstate baryon with the final-state baryon and the emitted photon, where the photon is a U-spin scalar with $U=0$, resulting in

$$
\begin{aligned}
\left\langle\Delta^{0} \mid n \gamma\right\rangle & =\langle 1-1 \mid 1-1 \quad 0 \quad 0\rangle=1 \\
\left\langle\Sigma^{* 0} \mid \Sigma^{0} \gamma\right\rangle & =\frac{1}{2}\left\langle\begin{array}{lllll}
1 & 0 \mid 0 & 0 & 0 & 0\rangle
\end{array}=\frac{1}{2} .\right.
\end{aligned}
$$

The ratio strictly based on this rotation is then

$$
\frac{\left|\left\langle\Delta^{0} \mid n \gamma\right\rangle\right|^{2}}{\left|\left\langle\Sigma^{* 0} \mid \Sigma^{0} \gamma\right\rangle\right|^{2}}=4
$$

The phase-space factors required for the radiative width are

$$
\Gamma_{\gamma} \propto \frac{M_{B}^{\prime}}{M_{B}} q^{2}\left|A_{B \rightarrow B^{\prime} \gamma}(q)\right|^{2},
$$

where $q$ is the center-of-mass momentum, $A$ is the amplitude of the decay (which contains a factor of $\sqrt{q}$ ), and $M_{B}$ $\left(M_{B^{\prime}}\right)$ is the mass of the decaying (final-state) hyperon. The ratio can then be expressed as

$$
\frac{\left\langle\Delta^{0} \mid n \gamma\right\rangle^{2}}{\left\langle\Sigma^{* 0} \mid \Sigma^{0} \gamma\right\rangle^{2}}=\left(\frac{M_{n}}{M_{\Delta}}\right)\left(\frac{M_{\Sigma^{0}}}{M_{\Sigma^{* 0}}}\right)^{-1}\left(\frac{q_{n}}{q_{\Sigma^{0}}}\right)^{3} 4=10.90 .
$$

The values for the center-of-mass momentum are $q_{n}=$ $0.259 \mathrm{GeV} / c$ and $q_{\Sigma^{0}}=0.178 \mathrm{GeV} / c[14]$.

This implies that the U-spin prediction for the partial width of the electromagnetic decay, using the measured width of the $\Delta^{0} \rightarrow n \gamma$, is

$$
\frac{\Gamma\left(\Delta^{0} \rightarrow n \gamma\right)}{10.90}=\frac{660 \pm 60}{10.90}=60.55 \pm 5.5 \mathrm{keV}
$$

where the partial width from the $\Delta \rightarrow n \gamma$ comes from ref. [14]. Finally the transition magnetic moment can be written as

$$
\mu_{\Sigma \Sigma^{* 0}}=\sqrt{\frac{2 M_{p}^{2} \Gamma_{\Sigma^{* 0} \rightarrow \Sigma^{0} \gamma}}{\alpha p_{\gamma}^{3}}}=(1.61 \pm 0.07) \mu_{N} .
$$

Similarly the U-spin prediction for the trasition magnetic moment for $\Sigma^{*+} \rightarrow \Sigma^{+} \gamma$ is $(3.22 \pm 0.15) \mu_{N}$ and for the $\Xi^{* 0} \rightarrow \Xi^{0} \gamma$ is $(3.21 \pm 0.15) \mu_{N}$. By neglecting the isospin symmetry breaking, the isospin-violating magnetic moment combinations give relations which can be used to determine six magnetic moments in terms of the others [9]. Consequently, one of the dependent magnetic moments in 
Table 1. Transition magnetic moments of the $\Sigma^{*}(1385)$ and $\Xi^{* 0}$ from the $1 / N_{c}$ expansion, the naive quark model (QM), and the U-spin prediction are compared with the experimental results. The $1 / N_{c}$ predictions are from the fit $E^{\prime}$ of [9]. All units are in $\mu_{N}$.

\begin{tabular}{crrcr}
\hline Transitions & $1 / N_{c}$ & QM $[16]$ & U-spin & Experiment \\
\hline$\mu_{\Sigma \Sigma^{*+}}$ & $2.96 \pm 0.04$ & 2.33 & $3.22 \pm 0.05$ & $3.22 \pm 0.45$ \\
$\mu_{\Lambda \Sigma^{* 0}}$ & $2.96 \pm 0.04$ & 2.28 & $2.68 \pm 0.04$ & $2.75 \pm 0.25$ \\
$\mu_{\Sigma \Sigma^{* 0}}$ & $1.34 \pm 0.04$ & 1.02 & $1.61 \pm 0.07$ & \\
$\mu_{\Sigma \Sigma^{*-}}$ & $-0.27 \pm 0.04$ & -0.30 & $0.0 \pm 0.20$ & \\
$\mu_{\Xi \Xi^{* 0}}$ & $2.96 \pm 0.04$ & 2.33 & $3.21 \pm 0.15$ & \\
\hline
\end{tabular}

the isospin symmetry limit can be used to estimate $\mu_{\Sigma \Sigma^{* 0}}$ using the U-spin predicted transition magnetic moments just acquired. The magnetic moment in the isospin symmetry limit is expressed as

$$
2 \mu_{\Sigma \Sigma^{* 0}}-\mu_{\Sigma \Sigma^{*+}}=\mu_{\Sigma \Sigma^{*-}}=(0.0 \pm 0.20) \mu_{N} .
$$

The experimental values in each case are shown in table 1 along with theoretical predictions from the $1 / N_{c}$ expansion, the naive quark model prediction, and the U-spin predictions. The $1 / N_{c}$ predictions in table 1 are obtained using the fit $E^{\prime}$ from [9], where the 10 most accurately measured magnetic moments were used in an 8 operator fit, resulting in a theorical uncertainty of $0.04 \mu_{N}$.

The deduced U-spin transition magnetic moments indicates a zero transition magnetic moment for the EM decay of the $\Sigma^{*-}$, whereas the prediction from the fit $E^{\prime}$ of [9] suggests a negative transition magnetic moment.

The two experimental values that exist agree with the $1 / N_{c}$ expansion predictions as well as the U-spin predictions. There is remarkable disagreement with traditional models as indicated with the naive quark model [16]. The $1 / N_{c}$ expansion predictions are not consistent with the the deduced U-spin predictions for the final three decays listed in the table. In fact for the $\Sigma^{*-} \rightarrow \Sigma^{-} \gamma$ the $1 / N_{c}$ expansion is consistent with the naive quark model. The U-spin value are based on the data from the $\Delta$ resonance and have phenomenological contributions which, to a large extent, are weighted out of the fits in the $1 / N_{c}$ expansion. Non-analytic chiral corrections are larger for baryon magnetic moments than for other baryon observables. These chiral corrections are mostly meson cloud effects.

The upper limit on the U-spin deduced decay width of $\Sigma^{*-} \rightarrow \Sigma^{-} \gamma$ is $0.91 \mathrm{keV}$. This is a much smaller upper bound than the one set by the SELEX results [17]. In lieu of an experimental branching ratio for the EM decay of either the $\Sigma^{*-}$ or $\Sigma^{* 0} \rightarrow \Sigma^{0} \gamma$, these results are the only ones available for comparison with theory. The CLAS analysis is presently taking place with an attempt to obtain accurate experimental results on these decays.

\section{$5 \mathrm{U}$-spin symmetry breaking}

An experimental observation of the $\Sigma(1385)^{-} \rightarrow \Sigma^{-} \gamma$ decay would give much needed information on the $S U(3)$ breaking mechanism. The observables are sensitive to $S U(3)$ flavor symmetry breaking and strangeness suppression. There has been much theoretical interest in the framework of the $S U(3)$ and $S U(6)$ symmetry-breaking schemes [18-20], as well as the Isgur and Karl nonrealivistic quark model [21], and the MIT bag model [22]. There are also prediction made with the Skyre model [15, 23,24], heavy baryon chiral perturbation theory [25, 26], quenched lattice QCD [27], a field theoretic quark model [28], and exchange currents emphasized chiral constituent quark model $[29,30]$, not to mention the predictions made in the $1 / N_{c}$ expansion [9]. All the theoretical work thus far has lead to radiative decay widths for the $\Sigma(1385)^{-} \rightarrow \Sigma^{-} \gamma$ in the range of $1-9.5 \mathrm{keV}$. Unfortunately the observation of the $\Sigma(1385)^{-} \rightarrow \Sigma^{-} \gamma$ decay would require very small experimental uncertainties to understand the relevant non-valence degrees of freedom in the effective quark-quark interaction. Nevertheless, the resulting U-spin predicted decay width presented here implies that the decay width value may be smaller than $1 \mathrm{keV}$.

Prior to the new $1 / N_{c}$ expansion, it was only the Uspin prediction [13] for the decay widths of $\Sigma^{*+} \rightarrow \Sigma^{+} \gamma$ and $\Sigma^{* 0} \rightarrow \Lambda \gamma$ that had comparable values to the experimental results. The table only lists the $1 / N_{c}$ predictions from the fit $E^{\prime}$ of [9] which do not include the experimental values listed in the table. There is an additional fit in ref. [9] which includes these new CLAS measurements and reduces the fit error which is listed as the fit $E$ in [9]. However, the values are consistent with the fit $E^{\prime}$ within the theoretical uncertainty $\sigma^{\text {theory }}$. The fits to many of the experimental magnetic moments are dominated by $\sigma^{\text {theory }}$. For fits $E$ and $E^{\prime}, \sigma^{\text {theory }}$ is large in comparison to the experimental errors for most of the octet magnetic moments, but significantly smaller than the experimental errors for the $\Delta^{++}, \Omega^{-}$and the decupletoctet transition magnetic moments. This implies the octet magnetic moments are dominating the fits. This results in fits $E$ and $E^{\prime}$ to yield very similar operator coefficients even though $\Delta^{++}$and the new CLAS data are not included in fit $E^{\prime}$. The non-analytic chiral corrections contained in these measurements is weighted as to not have an effect within the resulting $\sigma^{\text {theory }}$ on the strange sector transition magnetic moments.

It is for this reason that we present the U-spin predictions as an alternative to the $1 / N_{c}$ expansion until adequate lattice calculation can be made. The disagreement in past predictions with the experimental values could be due to the meson cloud effects not yet considered in any of the theoretical frameworks mentioned. The U-spin prediction for the $\Sigma^{*}$ radiative decays uses empirical information from the $\Delta$ radiative decay such that contributions from the meson cloud effect are inherent in the calculation. The meson cloud effect is thought to contribute on the order of $40 \%$ [31] but there has not yet been theoretical work to give the precise contributions. The zero transition magnetic moment for $\Sigma^{*-} \rightarrow \Sigma^{-} \gamma$ compared with the naive quark model and the $1 / N_{c}$ expansion is indicative of this contribution.

The upper limit on the degree of the $S U(6)$ symmetry breaking can be given using the deduced upper limit of 
the decay width of $\Sigma^{*-} \rightarrow \Sigma^{-} \gamma$ and the U-spin predicted width of $\Sigma^{*+} \rightarrow \Sigma^{+} \gamma$,

$$
\frac{\Gamma\left(\Sigma^{*-} \rightarrow \Sigma^{-} \gamma\right)}{\Gamma\left(\Sigma^{*+} \rightarrow \Sigma^{+} \gamma\right)}=\frac{0.91 \mathrm{keV}}{250 \mathrm{keV}}=0.4 \%
$$

Detailed calculations have been carried out by many groups $[19,32,33]$ and all come up with ratios that are of the order of a few percent. The lattice result is of particular interest, because the quarks are current quarks and have much different interactions with the photon than the simple quark model, yet the degree of symmetry breaking is still larger than the value presented here.

Perhaps the results presented here can help to serve as a motivation for the theoretical community to calculate the baryon magnetic moments with higher accuracy, both in the $1 / N_{c}$ expansion and on the lattice, both of which are based directly on QCD.

The authors thank the staff of the Thomas Jefferson National Accelerator Facility who made the experiment possible. This work was supported in part by the Chilean Comisión Nacional de Investigación Científica y Tecnológica (CONICYT), the Italian Istituto Nazionale di Fisica Nucleare (INFN), the French Centre National de la Recherche Scientifique, the French Commissariat à l'Energie Atomique, the U.S. Department of Energy, the National Science Foundation, the UK Science and Technology Facilities Council (STFC), the Scottish Universities Physics Alliance (SUPA), and the National Research Foundation of Korea. The Southeastern Universities Research Association (SURA) operates the Thomas Jefferson National Accelerator Facility for the United States Department of Energy under contract DE-AC05-84ER40150.

Open Access This is an open access article distributed under the terms of the Creative Commons Attribution License (http://creativecommons.org/licenses/by/3.0), which permits unrestricted use, distribution, and reproduction in any medium, provided the original work is properly cited.

\section{References}

1. F. Close, An Introduction to Quarks and Partons (Academic Press, London, 1979).

2. N. Isgur, G. Karl, Phys. Rev. D 21, 3175 (1980).

3. R.H. Dalitz, D.G. Sutherland, Phys. Rev. 146, 1180 (1966).
4. B. Julia-Diaz, T.-S.H. Lee, T. Sato, L.C. Smith, Phys. Rev. C 75, 015205 (2007).

5. D.B. Leinweber, T. Draper, R.M. Woloshyn, Phys. Rev. D 48, 2230 (1993).

6. C. Alexandrou et al., Phys. Rev. D 77, 085012 (2008).

7. H.-W. Lin, S.D. Cohen, R.G. Edwards, D.G. Richards, Phys. Rev. D 78, 114508 (2008).

8. E. Jenkins, A.V. Manohar, Phys. Lett. B 335, 452 (1994).

9. E. Jenkins, Phys. Rev. D 85, 065007 (2012).

10. C. Levinson,H. Lipkin, S. Meshkov, Phys. Lett. 7, 81 (1963).

11. CLAS Collaboration (D. Keller et al.), Phys. Rev. D 83, 072004 (2011).

12. B.A. Mecking et al., Nucl. Instrum. Methods A 503, 513 (2003).

13. CLAS Collaboration (D. Keller et al.), Phys. Rev. D 85, 052004 (2012).

14. Particle Data Group, J. Phys. G 37, 075021 (2010).

15. T. Haberichter, H. Reinhart, N.N. Scoccola, H. Weigel, Nucl. Phys. A 615, 291 (1997).

16. R. Dhir, R.C. Verma, Eur. Phy. J. A 42, 243 (2010).

17. Selex Collaboration (V.V. Molchanov et al.), Phys. Lett. B 590, 161 (2004).

18. H.J. Lipkin, Phys. Rev. D 7, 846 (1973).

19. H. Lipkin, M. Moinester, Phys. Lett. B 287, 179 (1992).

20. A. Akhiezer, M.P. Rekalo, JETP Lett. 1, 29 (1965).

21. J.W. Darewych, M. Horbatsch, R. Koniuk, Phys. Rev. D 28, 1125 (1983).

22. R.H. Hackman, N.G. Deshpande, D.A. Dicus, V.L. Teplitz, Phys. Rev. D 18, 2537 (1978).

23. C.L. Schat, C. Gobbi, N.N. Scoccola, Phys. Lett. B 356, 1 (1995).

24. A. Abada, H. Weigel, H. Reinhardt, Phys. Lett. B 366, 26 (1996).

25. M.N. Butler, M.J. Savage, R.P. Springer, Nucl. Phys. B 399, 69 (1993)

26. M. Napsuciale, J.L. Lucio, Nucl. Phys. B 494, 260 (1997).

27. D.B. Leinweber, T. Draper, R.M. Woloshyn, Phys. Rev. D 48, 2230 (1993).

28. R.K. Sahoo, A.R. Panda, A. Nath, Phys. Rev. D 52, 4099 (1995).

29. G. Wagner, A.J. Buchmann, A. Faessler, Phys. Rev. C 58, 1754 (1998).

30. G. Wagner, A.J. Buchmann, A. Faessler, J. Phys. G 26, 267 (2000).

31. T. Sato, T.-S. Lee, Phys. Rev. C 54, 2660 (1996).

32. V.V. Molchanov, Phys. Lett. B 590, 161 (2004).

33. D. Leinweber, T. Draper, R. Woloshyn, Phys. Rev. D 48, 2230 (1993). 